\title{
Sizing up Exotic Nuclei with Radioactive Molecules
}

\author{
New research shows that radioactive molecules can be used to study the \\ variation in the shapes and sizes of exotic nuclei that are particularly \\ sensitive to fundamental symmetry violations.
}

By Jaideep Taggart Singh

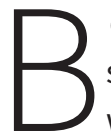
ecause matter and antimatter show near-perfect symmetry with respect to the laws of physics as we know them, physicists expect that equal amounts of matter and antimatter were present in the early Universe. But the concordance of ever more precise astronomical and cosmological observations indicates that the visible Universe is completely dominated by matter, with hardly any antimatter anywhere. What caused the preponderance of matter?

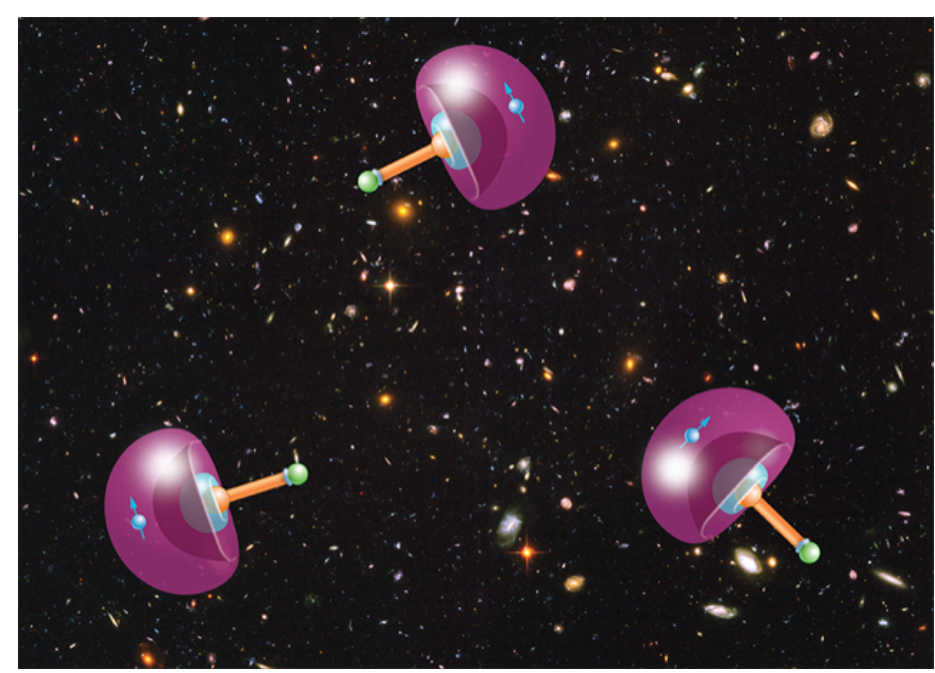

Figure 1: Studying certain radioactive nuclei within polar molecules, such as the radium nuclei in radium monofluoride, is a promising approach for understanding fundamental symmetry violations that may be responsible for why there is so little antimatter in our Universe.

Credit: APS/Carin Cain; NASA, ESA, S. Beckwith (STSCI), the HUDF Team
Physicists suspect that the answer to this question lies in fundamental symmetry violations, such as subatomic particle interactions that are not the same when the arrow of time is reversed $[1,2]$. One promising approach to finding these violations lies in studying certain pear-shaped radioactive nuclei within polar molecules (see Viewpoint: Designer Molecules for Fundamental-Symmetry Tests). Research has shown that the energy spectra of such molecules are exceptionally sensitive probes of symmetry violations $[3,4]$. To harness this discovery potential, researchers need to develop a better understanding of the energy spectra of these radioactive molecules. In particular, they need to quantify how the interactions between a molecule's nuclei and electrons influence the quantum-mechanical energy levels of the molecule. Silviu-Marian Udrescu of the Massachusetts Institute of Technology and co-workers have now addressed this question by measuring the quantum-mechanical energy levels of radium monofluoride (RaF) molecules with different $\mathrm{Ra}$ isotopes [5]. By taking into account the nuclear sizes of the various Ra isotopes, the team determined the isotope shifts of these energy levels and tested quantum chemical models used to predict molecular spectra [5]. This new approach will help researchers design symmetry violation tests with ever-increasing sensitivity using molecules with this and other heavy nuclei.

Testing symmetry violations with these molecules requires a detailed understanding of the molecular energy levels. Nuclei influence the energy levels of atoms through their interactions with the electrons. For heavy nuclei, the key factors affecting energy levels are the magnitude and variation of the electron density within the nuclear volume. Laser spectroscopic 
measurements of transitions between energy levels in atoms, when coupled with atomic theory, allow one to extract quantitative information about the shapes and sizes of nuclei in a way that is independent of nuclear models [6]. This technique provides a powerful test of nuclear structure theories, which aim to predict these properties. Changing the number of neutrons within the nucleus for a particular chemical element results in a small but measurable shift in the atomic energy levels, called an isotope shift. Measurements of isotope shifts in atoms for chains of isotopes have revealed the variations in the shape and size of atomic nuclei, as recent work showed in nobelium (see Focus: Laser Bags a Giant Nucleus) [7].

Although these isotope shifts are also observable in molecules, they haven't been measured in molecules containing nuclei heavier than lead until now.

Udrescu and co-workers have now measured isotope shifts in $\mathrm{RaF}$ for a series of Ra isotopes (with atomic weights of 223-226 and 228) using laser spectroscopy. By combining these isotope shifts with literature values for the size of Ra isotopes, the researchers extracted the isotope shift constant, a quantity that describes the influence of nuclear size on the isotope shift. They then compared the measured isotope shift constant to that derived from quantum chemical calculations. Since this constant is crucially sensitive to the overlap of the electronic quantum-mechanical wave function with the nuclear volume, this comparison with experiments provides a stringent test of quantum chemical theories.

The researchers produced $\mathrm{Ra}$ isotopes at CERN's Isotope Separator On-Line DEvice (ISOLDE) by bombarding a uranium target with high-energy protons [8]. The team formed $\mathrm{RaF}^{+}$ molecular ions by heating this target in the presence of carbon tetrafluoride gas. These molecular ions were subsequently extracted, mass-selected, cooled, bunched, and neutralized to form a pulsed beam of neutral RaF molecules containing a specific isotope of Ra. To make spectroscopic measurements of the energy transitions of interest, the researchers illuminated these neutral molecules with a laser whose frequency could be scanned through the molecular spectra. Those molecules that became resonantly excited were then ionized again with a second laser beam at a different, fixed frequency. An ion detector then counted the resulting RaF+ molecular ions as a function of the tunable laser frequency. The experimental isotope shift constant was finally derived by fitting a line to a plot of the measured shifts as a function of the known nuclear sizes of the Ra isotopes from the literature.

The researchers also calculated the isotope shift constant using relativistic molecular theory codes. Although the RaF molecule has just one valence electron, the researchers performed the calculations by taking into account the 17 outermost electrons and the correlations among them. They also carried out a limited set of more time-consuming and higher accuracy calculations using 27 correlated electrons and used these results to scale the results from the 17-electron calculations. The calculations were not overly sensitive to the exact shape of the nuclear charge distribution and agreed with experimental results to within $10 \%$. Furthermore, the team used the empirically determined isotope shift constants to deduce the difference in the effective electron density between the ground and excited states for the transition probed. This difference, which was also in good agreement with the quantum chemical calculations, is important because it largely determines the surprisingly large sensitivity of the molecular energy levels to nuclear size effects.

The results of this work indicate that the technique can measure molecular isotope shifts with sufficient precision to study the variation of nuclear shapes and sizes across the isotope chain of a heavy element. This conclusion has important implications for the study of the nuclear structure of exotic actinide nuclei, which may be more accessible in molecular form than as isolated atoms. In addition, the successful benchmarking of quantum chemical calculations against experimental data is particularly valuable, as the reliability of these calculations is key to understanding the electron-nucleus interactions that determine the sensitivity of radioactive molecules to violations of fundamental symmetries.

Certain radioactive pear-shaped nuclei, such as protactinium nuclei [9] and the Ra nuclei [10] that Udrescu and colleagues have studied, have exquisite sensitivity to fundamental symmetry violations [11]. This sensitivity can be further enhanced when these exotic nuclei are probed inside of polar molecules such as RaF. By increasing the laser spectral resolution, the researchers will be able to study nuclear-spin-related effects in $\mathrm{RaF}$ as well as other radioactive molecules such as thorium monoxide (ThO) and protactinium monoxide $(\mathrm{PaO})$. More precise measurements of molecular 
isotope shifts may also allow researchers to search for new types of exotic forces (see Synopsis: Hints of Dark Bosons).

Jaideep Taggart Singh: Facility for Rare Isotope Beams, Michigan

State University, East Lansing, MI, USA

\section{REFERENCES}

1. H. R. Quinn, "The asymmetry between matter and antimatter," Phys. Today 56, 30 (2003).

2. N. Fortson et al., "The search for a permanent electric dipole moment," Phys. Today 56, 33 (2003).

3. P. Yu and N. R. Hutzler, "Probing fundamental symmetries of deformed nuclei in symmetric top molecules," Phys. Rev. Lett. 126, 023003 (2021).

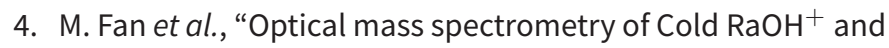
$\mathrm{RaOCH}_{3}^{+}$," Phys. Rev. Lett. 126, 023002 (2021).

5. S. M. Udrescu et al., "Isotope shifts of radium monofluoride molecules," Phys. Rev. Lett. 127, 033001 (2021).

6. P. Campbell et al., "Laser spectroscopy for nuclear structure physics," Prog. Part. Nucl. Phys. 86, 127 (2016).

7. S. Raeder et al., "Probing sizes and shapes of nobelium isotopes by laser spectroscopy," Phys. Rev. Lett. 120, 232503 (2018).

8. R. F. Garcia Ruiz et al., "Spectroscopy of short-lived radioactive molecules," Nature 581, 396 (2020).

9. J. T. Singh, "A new concept for searching for time-reversal symmetry violation using Pa-229 ions trapped in optical crystals," Hyperfine Interact. 240, 29 (2019).

10. L. P. Gaffney et al., "Studies of pear-shaped nuclei using accelerated radioactive beams," Nature 497, 199 (2013).

11. N. Auerbach et al., "Collective $T$ - and $P$-odd electromagnetic moments in nuclei with octupole deformations," Phys. Rev. Lett. 76, 4316 (1996). 